\title{
APROXIM ACIÓN AL DEBATE SOBRE LA MODERNIDAD EN AMÉRICA LATINA: PROYECTO DE LA MODERNIDAD Y APERTURAS POSTMODERNAS*
}

\author{
Por: Erna von der Walde
}

\section{A Rosario}

Tratar de delimitar en tan poco tiempo un tema tan complejo va a significar que incurriré en muchas simplificaciones y esquemas inevitables. Hay múltiples versiones de lo que es la modernidad, distintas cronologías, distintos enfoques. Adicionalmente, surge el problema de que quiero presentar algunas modalidades del debate sobre la modernidad en América Latina, pero realmente empiezo exponiendo puntos del debate europeo. Mi intención es partir de Habermas y Lyotard para luego referime a las modalidades que adopta el debate en América Latina, señalando en qué medida hay una apertura propiciada por el pensamiento posmoderno.

\section{¿Qué se entiende por modernidad?}

Para conservar una cierta coherencia me basaré en la que presenta Jurgen Habermas en un ensayo de 1981, titulado "Modernidad vs postmodernidad» ${ }^{1}$. Aquí se postula básicamente que aunque desde aproximadamente el siglo $\mathrm{V}$ de nuestra era, se ha venido utilizando el término «modemo» para diferenciarse de un momento pasado que se considera superado, hasta el siglo XVIII este uso siempre ha sido referido a lo anterior, al pasado, a la antigüedad. Es a partir de la época de la llus tración que cambia nuestra conciencia de ser modemos, pues es to se proyecta ya no hacia el pasado sino hacia el futuro. Es decir, que la modernidad es también aquello que va vinculado con lo que Néstor García Canclini, en el primer capítulo de Culturas híbridas: Estrategias para entrar y salir

\footnotetext{
* Texto de la conferencia leída en el Departamento de Lenguas de la Universidad Pedagógica Nacional, Bogotá, Abril 27, 1994.

1Jurgen Habermas, "Modernidad vs postmodernidad", en: Fernando Viviescas y Fabio Giraldo Isaza (Comps.). Colombia: el despertarde la modernidad. Bogotá: Foro Nacional por Colombia, 1991.17-31. (Aquí me basaré en esta versión del artículo.)

La versión española de este artículo de Habermas es tomada de la versión inglesa, que apareció por primera vez en New German Critique, 22 (invierno 1981). 3-14, y luego en Hal Foster (ed.). The Anti Aesthetic. Port Townsend, Washington: The Bay Press, 1984. (Este libro a su vez fue reeditado con el título Postmodern Culture. Londres: Pluto Press, 1985.) Esta es a su vez una versión recortada de la conferencia que dictó Habermas en Frankfurt cuando se le conœdió el premio Adorno de esta ciudad bajo el título de 《<Die Moderne - em unvollendetes Projekt>> (en: Jürgen Habermas. Die Moderne - em unvollendetes Projekt. Philosophischpolitische Aufsätze 1977-1990. Leipzig: Reclam, 1990.32-54. Otras traducciones españolas de este artículo de Habermas traduœen el título alemán pero se basan también en la versión inglesa. Ver Jürgen Habermas. "Modernidad, un proyecto incompleto", en Nicolás Casullo, ed. El debate modernidad/ postmodernídad, Buenos Aires: El cielo por asal to, 1993. 131-144.
} 
de la modernidad ${ }^{2}$ llama los cuatro proyectos de la modernidad, proyectos que tienen su origen en la llustración francesa y su consecuente revolución política. Estos cuatro proyectos son: un proyecto emancipador que implica la secularización de las ciencias y el arte, y su autonomía con respecto al poder, bien sea este religioso o político; un proyecto expansivo que implica la expansión y difusión del conocimiento, pero también la expansión del capital al ser aplicados estos conocimiento a la técnica y el desarrollo industrial; un proyecto renovador que implica la constante innovación, una exigencia que se les hace tanto a las ciencias y al arte como a los productos de consumo; y por último un proyecto democratizador que implica la difusión extendida de los saberes para lograr una evolución racional y moral.

A la noción de modernidad van unidas las nociones de progreso, desarrollo, evolución y sobre todo de un cierto tipo de racionalidad, a la que se subordinan las otras. La proyección hacia el futuro implica planear, pero también que es posible mejorar las condiciones actuales. Todas estas nociones le dan un enome peso valorativo a lo nuevo, a la innovación, que se vincula directamente con la idea de mejoramiento y crea una temporalidad lineal: de lo viejo a lo nuevo es progresar de lo menos bueno a lo mejor. Bajo esta concepción, el pasado es algo que se supera, lo tradicional queda relegado a ser aquello que tranca o retrasa el desarroIlo. Esta linealidad evolutiva, que tiene su origen en gran parte en la racionalidad de las ciencias naturales ${ }^{3}$, es el punto central de lo que podemos llamar la ideología modernizadora.

Ahora bien, desde que hay una conciencia de esa modernidad ha habido aproximaciones críticas a ella. Se considera que es Kant el primer filósofo en postular, entre otras, la racionalidad que cubre la modernidad, y en separar ésta en tres campos autónomos: el científico, o sea la razón pura; el moral, o sea la razón práctica; y el estético, que corresponde al campo más subjetivo del juicio. Después de Kant viene Hegel, quien dentro de la lógica que impone la misma racionalidad mo(lema ve sin embargo las implicaciones y contradicciones que ésta conlleva: a mayor expansion del individuo hay mayor disolución de la comunidad; plantea la necesidad de un Estado que controle las distintos conflictos que surgen del sistema que se va imponiendo, etc. Es muy amplia y compleja la filosofía de Hegel, pero sí podemos anotar que es tal vez el filósofo que planteó los problemas sustanciales que siguen debatiéndose alrededor de la modernidad ${ }^{4}$.

${ }^{2}$ Nestor García Canclini, Culturas hibridas: Estrategias para entrar y salir de la modernidad, México:

Grijalbo, 1990.p. 31-32.

${ }^{3}$ Ver Mary Louise Pratt. Imperial Eyes. Travel Writing and Transculturation. Londres: Routledge, 1992. En este magnífico libro Pratt muestra, entre otros, cómo el discurso de las ciencias naturales, particularmente la botánica y el sistema clasificatorio de Lineo, pero también la medicina, contribuye a la constitución de un discurso imperialista que los europeos utilizarán para intemretar las culturas extraeuropeas y justificar su proyecto expansionista.

${ }^{4}$ Ver Perry Anderson. "The Ends of History". En A Zone of Engagement. Londres: Verso, 1992. 279375. p.322., en donde cita a Kojéve: 'It there was from the beginning a Hegelian Left and a Hegelian Right, that is all there has been since Hegel.' History had unfolded within the categorical framework 
Sin duda la crítica más influyente has ta ahora a los conflictos que implica la modernidad ha sido la de Carlos Marx. Muchos de sus puntos son ya un lugar común en nuestra manera de entender la modernidad: el desarraigo que implican las nuevas estructuraciones sociales generadas por los nuevos modos de producción, sus ataques a la lógica del capital que, a la vez que implica la expansión económica, acarrea la pauperización de grandes sectores de la humanidad, los círculos viciosos de superproducción y desempleo, etc., observaciones que son mas que económicas porque implican esa sensación de que "todo lo sólido se desvanece en el aire», la que luego Marshall Bemann recogerá como título de su libro sobre la modernidad.

\section{EL DEBATE EN EUROPA}

¿En qué se diferencia entonces este nuevo debate sobre la modernidad de lo que se venía haciendo hasta fines de la década de los setentas? Para ponerlo en pocas palabras se trata de un cuestionamiento no ya de los problemas que acarrea la modernidad, asumida siempre como inevitable, sino de un cuestionamiento a la lógica misma, a la racionalidad que abarca el proyecto moderno.

Siendo un poco esquemática, podría decir que hay dos líneas o tendencias muy marcadas en el debate actual. La una sería la que surge en Alemania, encabezada por Habermas, y que podríamos llamar la de los defensores del proyecto de la modernidad. La otra sería la que surge en Francia, a cuya cabeza se ubica en el momento Lyotard, pero que participa del pensamiento de Foucault, Barthes, Derrida, y que podríamos llamar la de los que cuestionan dicho proyecto, pero que se conocen con el nombre algo equívoco de postmodemos a partir de la obra que publicó Lyotard en 1979, La condición post moderna. ${ }^{6}$

the German philosopher had conceived, even it its exact outcome was still uncertain. 'It cannot be said that history has refuted Hegelianism. At most one can say that it has still not arbitrated between "left" and "right" interpretations of Hegel's philosophy."' ["Si desde el principio hubo hegelianos de izquierda y hegelianos de derecha, también se puede decir que eso es todo lo que ha habido después de Hegel." La historia se había desplegado dentro del marco categórico que este filósofo alemán había concebido, aun cuando su resultado exacto fuera todavía incierto. "No se puede decir que la historia haya refutado el hegelianismo. Como máximo se puede decir que no ha arbitrado entre las interpretaciones de izquierda' y las de 'derecha' de la filosofía de Hegel."]. Más adelante anota Anderson respecto a Habermas y su crítica a Hegel: “... [H]is work [Hegel's] posed all íts [modernity's] basic problems at such a depth that Habermas insists - echoing Kojéves dictum forty years earlier - we still remain contemporaries of the Young Hegelians." p. 328 [EI primer teórico de a modernidad no logró, pues, serle fiel. Pero su obra planteó todos los problemas básicos con tal profundidad que Habermas insiste en que -haciendo eco al veredicto de Kojéve cuarenta años antes- seguimos siendo contemporáneos de los jóvenes hegelianos.]

${ }^{5}$ Como curiosidad vale a pena anotar que esta expresión en castellano es tomada de la traducción inglesa del Manifiesto del Partido Comunista y no de la castellana. El original alemán no tiene tampoco la capacidad sugerente de la frase inglesa.

JJean Francois Lyotard, La condición posmodema, Madrid: Ediciones Cátedra, 1986. Ver también del mismo autor La postrnodernidad (explicada a los niños), Barcelona: Gedisa, 1987. 


\section{HABERMAS}

¿En qué consiste la crítica que hace Habemas a la racionalidad en la que se fundamenta el proyecto modemo? Este pensador considera que esta racionalidad no es tina sino que ha devenidlo en el proceso de afianzamiento de la modemidad en dos racionalidades distintas. La una es la que él llama razón instrumental; a la otra la denomina razón comunicativa. La razón instrumental es aquella que vinculamos más bien con el proceso de modernización económica y social, que es la que rige en la industria, en la burocracia estatal. La razón comunicativa es aquella que organiza, por así decirlo, las otras áreas del vivir y el convivir, lo que Habemas llama mundos de vida, yen términos generales podemos llamar cultura entendida como aquello que «proporciona significado a la experiencia humana, seleccionándola y organizándola. Se refiere con amplitud a las formas por las que la gente da sentido a su vida, y no a la ópera o a los museos de arte» ${ }^{7}$ para decirlo en las palabras del etnólogo chicano Renato Rosaldo, formas que están tan arraigadas que se confunden con lo natural.

Habermas considera que el problema fundamental de nuestra condición moderna actual es la forma cómo la racionalidad instrumental va penetrando las formas de vida. En el ensayo que mencioné plantea además el problema de que el arte, sometido, por así decirlo, a los imperativos de renovación constante propios de la modernidad, se ha ido alejando cada vez más de estos mundos de vida y se ha ido convirtiendo en un área a la que sólo tienen acceso los especialistas. La propuesta de Habermas es que en lugar de prodamar la muerte del arte o de esperar del arte que busque un acercamiento a la vida, que rompa con la distinción entre arte y vida como en los surrealistas, es necesario por un lado seguir dejando el espacio abierto para la experimentación artística pero permitir que la experiencia del arte no sea algo dictaminado por los expertos: "Tan pronto como una experiencia tal se usa para iluminar una situación de una historia vital y se relaciona con problemas de la vida, entra en un juego de lenguaje que ya no es el de la crítica es tética.» ${ }^{8}$

Habemas, en suma, plantea que el proyecto moderno no se ha agotado como algunos proclaman, sino que es un proyecto incompleto que hay que revitalizar en parte des de el arte pero sobre todo desde los mundos de vida.

\section{LOS POSMODERNOS.}

Es muy complejo entrar a diferenciar las distintas posturas que hay dentro de lo que se ha denominado el postmodernismo. Por eso, al igual que con la otra tendencia me limitaré a presentar algunos puntos del pensamiento de Lyotard. El

\footnotetext{
${ }^{7}$ Renato Rosaldo, Cultura y verdad: Nueva propuesta de análisis social, México: Grijalbo, 1991, p. 35.

${ }^{8}$ op. cit. p. 28.
} 
discurso dominante puede decirse que se caracteriza por un cuestionamiento, en primera instancia, de lo que se ha denominado los metarrelatos.

¿Qué son los metarrelatos? Muy breve y esquemáticamente se puede decir que son aquellos sistemas de pensamiento que desde una sola lógica pretenden dar razón de toda la realidad, sometiendo todos los fenómenos a la coherencia del sistema. Según Lyotard, quien fue el que formuló la crítica de la modernidad en esos términos, " ${ }^{9}$ el pensamiento y la acción de los siglos $\mathrm{XIX}$ y $\mathrm{XX}^{10}$ están regidos por una Idea (entiendo Idea en el sentido kantiano del término). Esta Idea es la de la emancipación y se argumenta de distintos modos según eso que llamamos las filosofías de la historia, los grandes relatos bajo los cuales intentamos ordenar la infinidad de acontecimientos: relato cristiano de la redención de la falta de Adán por amor, relato aufklarer de la emancipación de la ignorancia y de la servidumbre por medio del conocimiento y el igualitarismo, relato es peculativo de la realización de la idea universal por la dialéctica de lo concreto, relato manxista de la emancipación de la explotación y de la alienación por la socialización del trabajo, relato capitalista de la emancipación de la pobreza por el desarrollo tecnoindustrial. Entre estos relatos hay materia de litigio, e inclusive materia de diferendo. Pero todos ellos sitúan los datos que aportan los acontecimientos en el curso de una historia cuyo término, aun cuando ya no quepa esperarlo, se llama libertad universal, absolución de toda la humanidad.» ${ }^{11}$

El pensamiento posmoderno considera que el discurso de la modemidad funciona con un dualismo que postula siempre al otro a partir de sí mismo y basado en una lógica que pone como lo otro, lo excluyente todo aquello que no cabe dentro de su coherencia discursiva. Pero simultáneamente se postula como universal.

El postmodernismo parte no de lo único, lo universal, sino más bien de la diferencia. Rompe con los discursos centrales en la medida en que señala que no es más que uno posible, que convive con muchos otros discursos. Señala además cómo convive este discurso con lo que creyó era lo otro, cómo eso otro foma parte de sí mismo, es a partir de lo que se cons tituye.

Se puede decir que hay muchos postmodernismos. Hay una versión facilista que partiendo de la postulación de la diferencia pretende que la crisis que eso implica en cuanto a valoraciones tiene la ventaja de que todo es permitido. Según esto ya no hay que establecer si una obra de arte es buena o mala, tampoco importa cómo se inscribe en una tradición de escritura o de lectura, cualquier criterio que se quiera puede ser tan válido como el otro.

\footnotetext{
${ }_{9}^{9}$ En La condición posmoderna.

${ }^{10}$ Los mismos siglos para los que en la cronología de Habermas se puede hablar de modernidad en el mismo sentido que la entendemos hoy.

${ }^{11}$ Misiva sobre la historia universal. En La Postmodernidad (explicada a los niños). p. 36. Ver también en el mismo libro "Apostilla a los relato"p. 29.
} 
Este tipo de postmodernismo tiende a descontextualizar y sacar de su entomo histórico y cultural los procesos de producción simbólica y termina haciéndole el juego a un neoconservadurismo político que cree que la crisis de la modemidad está en la cultura disoluta que corrompe la ética de trabajo en la que se basa el espíritu del capitalismo. ${ }^{12}$

Otra postura es la postura de pensadores como Lyotard. Este incorpora entre otras una critica del lenguaje que ya tiene tradición en Francia y que se puede comprender básicamente que consiste en un cuestionamiento de cómo construimos lo real a partir del lenguaje. Esta crítica viene inicialmente de la literatura, enfrentándose al realismo literario y desenmascarándolo como una forma que se pos tula como neutra y objetiva aunque es un constructo de lenguaje como cualquier otro. Para Lyotard la cris is de la modernidad cons is te en la perdida d parámetros, de un discurso único que le de coherencia a nuestra existencia.

Y si la modemidad se auto comprendía a entre otras a partir de un discurso úni$\mathrm{co}$, o metarrelato, la crisis de estos metarrelatos, el cuestionamiento al eje racional mismo del proyecto modemo nos permite pensar que estamos ante una condición «posmoderna». Esta crisis de los metarrelatos implica también un fin de las utopías, en el sentido de que si se cuestiona esa linealidad temporal del progreso y la evolución, tampoco queda muy claro cuál es el futuro ideal, utópico, al cual se aspira. Estas utopías, o teleologías ${ }_{13}$ los estadios finales a alcanzar, rigen por supuesto la lógica de los metarrelatos.

Ahora bien, el mismo Lyotard insiste en que la Postmodemidad no es un tiempo histórico, una nueva etapa que sucede a la modernidad. Es más bien una lectura distinta de esa modemidad, que si bien siempre tuvo críticas, nunca se postularon por fuera del discurso mismo que ésta establecía. Y se extiende a una crítica a los discurs os que, según él, a la vez que pretenden ser los que llevan a cabo el proyecto de la modernidad son realmente quienes lo han aniquilado. Tal es el caso de la tecnociencia capitalista, cuya caricatura macabra sería, por supues to, Auschwitz, pero también Vietnam y la Guerra del Golfo. ${ }^{14}$

12 Ver Habermas op.cit. y Daniel Bell. The Cultural Contradictions of Capitalism. Nueva York:
Harper Collins, 1978. (Hay traducción española: Las contradicciones culturales del capitalismo.)
Además ver la crítica de Andreas Huyssen a Habemas y la vinculación que hace este entre el
neoconservadurismo norteamericano, cuya figura principal es Bell, y el pensamiento
posestructuralista y posmodemo de los fraceses, en Andreas Huyssen."Guía del postmodernismo".
En Nicolás Casullo (ed.).266-318.Y en general, este ensayo de Huyssen es realmente una guía
para entender los vínculos y las diferencias entre muchas tendencias vanguardistas y
posmodernistas en el arte, pero también para diferenciar posiciones al respecto.
13 "Esto metarelatos no son mitos en el sentido de fábulas (induso el relato cristiano) [...] A
diferencia de los mitos, estos relatos no buscan la [..] Legitimidad en un acto originario fundacional,
sino en un futuro que se ha de producir, es decir en una Idea a realizar. Esta Idea (de libertad, de
"luz", de socialismo, etc.) posee un valor legitimante porque es universal. Como tal orienta todas las
realidades humanas, da a la modernidad su modo característico; el proyecto, ese proyecto que
Habermas considera aún inacabado y que debe ser retomado, renovado." Lyotard. La
Postmodernidad (explicada a los niños). p. 29-30.

${ }^{14}$ Ver Edward W. Said. Culture and Imperialism. Londres: Vintage, 1994. Sobre todo en la Introducción señala Said, que no tiene nada de posmoderno, cómo los Estados Unidos e Irak, cada uno a 


\section{EL DEBATE}

¿En qué consiste entonces el debate de la modernidad? Tal vez aquí sea necesario referirse al hecho de que el medio académico europeo y norteamericano (y este es al fin y al cabo un debate académico), es mucho más especializado. Aquí se trata casi que de un debate entre filós ofos sobre cuestiones de estética. Pero también es importante mencionar que en la concepción «modema» del arte, en lo que se han llamado los modernismos y las vanguardias, se considera que hay un vinculo entre el proyecto estético y el social. De hecho, el concepto de «postmodernismo» se utilizó por primera vez, en sentido más o menos análogo al actual, en la teoría de la arquitectura ${ }^{15}$ para oponer las tendencias arquitectónicas de la segunda postguerra europea a las modernistas que habían primado de 1910 a 1945. Paolo Portoghesi considera que lo característico de esta arquitectura posmoderna es su ruptura con la geometrta eudidiana -- es decir un cambio técnico dentro del campo de la arquitectura misma. Para Victorio Grigotti la fisura es más fundamental: se trata de la dislocación entre el proyecto social y el arquitectónico que venían vinculados en los movimientos modernistas como la Bauhaus. La arquitectura posmoderna sólo quiere generar pequeñas transformaciones en el espacio, y abandona el proyecto de transformación de todo el espacio humano. Es decir aquí estamos ante el postmodemismo como una tendencia artística que reacciona ante la tendencia dominante hasta ese momento: el modemismo. Pero también se establece una relación entre un movimiento artístico y una condición social. ${ }^{16}$

En la discusión euro-norteamericana se puede ubicar, por un lado, una discusión sobre la condición de la modernidad en la etapa del capitalismo postindustrial, por otro un debate sobre un arte que se ha desligado de los proyectos sociales. Pero también hay autores que buscan establecer los nexos entre una y otra.

En términos institucionales académicos el debate se puede describir como una polémica entre el pensamiento social (de los alemanes, pero también de los ingleses) y las tendencias del posestructuralismo francés. Esta polémica tiene de llamativo, como anota Scott Lash, que se establece un diálogo entre los alemanes y los franceses, quienes venían trabajando en los últimos veinte años haciendo caso omiso unos de otros. ${ }^{17}$ Obviam ente lo expuesto aquí deja por fuera muchos

su manera, legítima a través de un "metarrelato" y una interpretación de su propio pasado la "necesidad" de su intervención.

${ }^{15}$ En la literatura hispanoamericana ya Federico de Onís había utilizado el témino

"postmodernismo" para referirse a la generación de poetas que vino después de los modernistas. Ver Federico de Onís. Antología de la poesía española e hispanoamericana (1882-1932). Madrid: Casa Editora Hernando, 1934.

${ }^{16}$ Me baso aquí en lo que dice Jean Francois Lyotard en "Defining the Postmodern . En Lisa Appignanesi (ed.). Postmodernismo. ¡CA Documents. Londres: Free Association Books, 1989. 710. p. 7.

${ }^{17}$ Scott Lash. "Postmodernidad y deseo (Sobre Foucault, Lyotard, Deleuze, Habermas)... En Nicolás Casullo (comp.). 357-400. 
otros elementos de la discusión: un punto central que no he tocado pero que es fundamental es la cuestión de la cultura de masas. En los textos que expuse se discute el proyecto de la modernidad o su agotamiento desde los impulsos de ésta en el modernismo, en su manifes tación simbólica.

Pero la cultura de masas se ha concebido como el "otro» de la cultura de la modernidad y en este debate entra también a cuestionarla. ${ }^{18}$

En Estados Unidos existe una variante que incluye también puntos de la discusión europea y en la que las teorías de algunos, como Derrida y Foucault, desempeñan un papel importante. Se trata de la polémica en torno a las humanidades en la que los distintos grupos «minoritarios» (mujeres, homosexuales, negros, his panos, as iáticos, musulmanes, etc.) han cuestionado el carácter "eurocéntrico, androcéntrico, heterosexual y etnocéntrico" de la cultura oficial. De por sí mucho del cuestionamiento al canon oficial de la cultura y al discurso de poder dominante proviene de críticos que pertenecen a grupos «minoritarios», el «otro» de la cultura oficial. ${ }^{19}$

Vale la pena anotar aquí que, de hecho, una gran diferencia entre la discusión que se da en Europa y la que se da en los Estados Unidos, es que en la primera son pocos, si es que los hay, ${ }^{20}$, los miembros de grupos marginales o minoritarios que entran a participar en la discusión. Si bien se teoriza sobre el otro (Foucault), se habla de los derechos del otro (Lyotard), cabe preguntarse si esto no sigue

\footnotetext{
${ }^{18}$ Ver Andreas Huyssen. After the Great Divide.Modernism. Mass Culture, Pos tmodernism. Bloomington e Indianapolis: Indiana University Press. 1986. También Gianni Vattimo."Postmodernidad: ¿una sociedad transparente?.En Viviescas y Giraldo (comp.). 188196. y Jean Baudrillard. "The Ecstasy of Communication”. En Hal Foster (ed.) 126-134.

${ }^{19}$ Ver Craig Owens. "The Discourse of Others:
}

Feminism and Postmodernism". En Hal Foster. Postmodern Culture. 57-82. El caso ejemplar es sin duda Foucault, auyo homosexualismo no es ningún secreto. Entre los autores que he citado y mencionado aquí se encuentran casos de representantes de "minorías" como Edward W. Said, palestino norteamericano o Renato Rosaldo, chicano. Gayatri Spivak, feminista hindú residente en los Estados Unidos, ha hecho importantes contribuciones al questionamiento de Marx, Freud y el discurso imperialista. Ver Gayatri Chakravorty Spivak. In Other Wollds. Essays in Cultural Politics. Londres: Methuen, 1987. Ella se ubica, con otros provenientes de las antiguas colonias inglesas como Homi Bhabha y Said, en el área que se ha llamado estudios poscoloniales en el que se están debatiendo auestiones culturales en relación con globalización, fragmentación y nuevas formas de imperialismo.

${ }^{20}$ Pareciera que me contradigo habiendo afirmado hace apenas unas líneas que Foucault si pertenece a uno de esos grupos marginales, el de los homosexuales. El hecho es que este grupo es marginal en téminos de ciertos derechos, y de una concepción burguesa de la familia y la sexualidad. Pero culturalmente participan de la cultura oficial, y en términos de estructuras de poder (punto fundamental del pensamiento de Foucault) no son necesariamente marginados, o no lo son necesariamente por razón de su sexualidad. Una aguda y pertinente crítica a la visión de Foucault sobre cuestiones del así Ilamado Tercer Mundo es la de Spivak. "Can the Subaltern Speak?. En Lary Nelson y Lawrence Grossberg (eds.). Marxism and the Interpretation of Culture. Urbana, Chicago: University of Illinois Press, 1988. 271 -313. 
siendo parte del gesto euro centrista y moderno que postula lo europeo como el ser y lo extra-europeo como lo otro. ${ }^{21}$

\section{El debate en América latina}

La década de los ochenta en América Latina empieza bajo un signo muy distinto al que rige en Europa y en Estados Unidos. Hasta ahora me he referido muy poco a las circunstancias sociales, económicas y políticas en las que se da este debate. No podemos olvidar que la discusión europea se da en la década del consumismo, los a yuppies, el boom económico de lo que se ha venido a llamar la era ReganThatcher ( $\mathrm{y}$ hasta cierto punto Kohl). La condición posmodema de Lyotard es e de 1979. Y que esa misma década en y América Latina es la que se llama la "década perdida», en la que las economías nacionales de la mayoría de los países o no tuvieron mayor crecimiento, o decrecieron notablemente. La deuda externa creció, como era de suponerse. Pero simultáneamente se fueron implantando do las políticas económicas neo-liberales a todo lo largo y ancho del continente. Como dijera Carlos Monsiváis cuando unos estudiantes en Frankfurt el le preguntaron qué opinaba sobre la aplicación de éstas a la economía mexicana, «México lindo y querido, tan cerca de la sociedad de consumo y se tan lejos del poder adquisitivo». La brecha social se fue agrandando. Del hedonismo del consumo que se supone sea la característica de la sociedad post-industrial que proclaman ciertos postmodernismos es muy poco lo que pueden participar los latinoamericanos, los africanos, los as iáticos o los europeos orientales. Es decir, la idea de una sociedad post-industrial, en donde las masas trabajadoras se concentran en el sector terciario, más de servicios que de producción de bienes, una sociedad individualizada porque las transformaciones telemáticas hacen prescindible toda acción comunitaria, en donde el Estado pasa a ser tan sólo responsable de proveer cierta infraestructura y de velar por un cierto orden, para que entre el mercado a ser el regulador de todas las otras instancias sociales, tal sociedad, pienso, es concebible o mejor dicho era concebible en una especie de fortaleza que es lo que era Europa occidental hasta 1989.

No deja de ser notorio que en estos últimos cuatro años es muy poco lo que se discute la tal sociedad posmoderna. Pero la izquierda, los defensores del proyecto de la modemidad, también callan. Pues, es fácil dejar que el mercado regule todo cuando de todas maneras ya todo está controlado. ¿Pero qué hacer cuando a los países más ricos entran diariamente cientos de miles de personas ofreciendo mano de obra barata? Evidentemente has ta ahí llegan las bondades del mercado. El Estado con mano fuerte interviene, cambia la constitución, le cierra la puerta a todo extranjero. La actitud entre nihilista y juguetona de los postmodernismos facilistas con su «anything goes», deja de funcionar ante el surgimiento de tanto grupo radical de derechas, que no sólo participa en acciones violentas sino que va

\footnotetext{
${ }^{21}$ Para una discusión de discursos sobre el otro que vienen postulados desde Europa ver Nelly Richard. "Periferias culturales y descentramientos postmodernos (Marginalidad Iatinoamericana y recompaginación de los márgenes). Punto de vista, 40 (XIV), 1991. 5-6.
} 
ganado curules parlamentarias en tantos países como es el caso del Frente nacional de Le Pen en Francia, los Republicanos alemanes y la Lega Norte italiana.

¿Desde la izquierda se prodama la sociedad multicultural, pero qué hacer cuando son tantas, tan diversas y se juntan en tan poco tiempo todas esas culturas? ¿Cómo evitar los nacionalismos basados en nociones de raza, Volk, etc, y sin embargo entrar a defender la propia nación? ¿Cómo compaginar el interés individual y también nacional con el ideológicamente más correcto de una Comunidad Europea, pero que temina siendo, como dice un dicho alemán, cortar la rama en la que uno está sentado?

En esa misma década América Latina tiene otros problemas. Se presencia el gradual fin de las dictaduras militares que fueron la marca característica del continente en la década del 70. En 1982 cae la dictadura argentina, le sigue algo) después la uruguaya y por último la chilena. Esto en cuanto al cono sur. En los países así llamados democráticos se observan variantes neo-populistas como la de Alan García en el Perú. A la izquierda no le va mejor que a la derecha militar. El régimen sandinista no sobrevive la presión de los embargos económicos y permanentes ataques militares conducidos por los Estados Unidos. De Cuba mejor no hablemos.

Si vamos a hablar del fin de las utopías, son muy distintas las utopías que finalizan para sociedades en donde impera el orden social para la gran mayoría, en donde a pesar de la recesión económica el $90 \%$ de la población goza de un bienestar que $80 \%$ de la población mundial ni siquiera logra concebir, agobiada como está por el hambre y la miseria. Es decir, que los problemas a los que se enfrenta la modernidad europea y norteamericana son muy distintos a los que afectan a los países de América Latina.

La primera pregunta que se plantea aquí es: ¿Hay modemidad en d América latina? Uno de o los problemas de abordar g esta pregunta es que tradicionalmente en nuestro continente la cuestión de la modemidad se ha formulado desde la modernización socioeconómica. Como anota el chileno José Joaquín Brunner, «la sociología y el d pensamiento crítico latinoamericanos han estado preocupados sobre todo de la modernización, o sea, del proceso de transición y cambio en las es tructuras lo de la economía y la sociedad $"{ }^{22}$

Ya me referí a lo que se entiende por modernidad partiendo de Habermas. Y en el texto ya mencionado este autor establece una relación entre modernidad y modernismo, es decir entre el fenómeno global y sus manifestaciones simbólicas. La modemización socio-económica es la que se orienta por la razón instrumental que se encuentra invadiendo los mundos de vida. Pero esta noción de la modernidad como el fenómeno amplio que abarca tanto la modernización socio-

22 José Joaquín Brunner. "Experiencias de la modernidad". En ibid. América Latina: Cultura y modernidad. México: Grijalbo, 1992.73-1 19. p.87. 
económico (orientada por una razón instrumental según Habemas), como la manifestaciones modernistas (o vanguardistas) en el arte, pero sobre todo una conciencia de ser moderno (que se postula a partir de la orientación hacia el futuro) pasa por otra obra: me refiero a Todo lo que es sólido se desvanece en el aire de Marshall Bermann. ${ }^{23}$

Esta obra es demasiado amplia y e densa para poderla comentar aquí. Me limitaré a exponer únicamente un punto fundamental que es la diferenciación entre modernización como el proceso socio-económico de la modernidad, el modernismo como las manifestaciones simbólicas y la modernidad, como «experiencia vital - experiencia del espacio y el tiempo, del ser y de los otros, de las posibilidades y los peligros de la vida» compartida por todos los hombres y mujeres del mundo.

Tomando esta noción de modemidad como la presenta Berman es evidente que en América Latina sí ha habido modernidad y que «hemos tenido un modernismo exuberante con una modemización deficiente» ${ }^{25}$. Pero le adicionalmente que esa experiencia vital si bien es compartida por todos los hombres y mujeres del mundo lo es de maneras distintas.

Ahora bien, la noción de modernidad, entendida desde la modernización, acompañada de la idea de progreso y de políticas económicas de modemización basadas en el desarrollo, adopta la modernidad euro-norteamericana como modelo y meta a alcanzar. De ahí a concluir que la diferencia que nos distingue es la del atraso no hay sino un paso. Pero es justamente en el área de la cultura que, como dice Jesús Martín-Barbero podemos notar que hay una «diferencia que

\footnotetext{
${ }^{23}$ Marshall Berman, Todo lo sólido se desvanece e el aire. La experiencia de la modernidad. México Siglo XXI, 1989. Este libro sirve directamente d inspiración a Beatriz Sano para su obra sobre 1 modernidad en Buenos Aires: Una modernidad periférica: Buenos Aires 1920 y 1930, Buenos Ai res: Ediciones Nueva Visión, 1988. Me atrevo sugerir aquí que una de las razones por la cual 1 obra de Berman ha tenido una recepción tan im portante en América Latina es el hecho de que re coge dentro del mismo gesto moderno tanto Goethe, Marx y Baudeíaire, como a lo que él mismo llama "el modernismo del subdesarrollo", de San Petersburgo. Es decir, Berman parece pode incorporar dentro de su análisis lo que siempre s consideraba lo no moderno, la periferia de la modernidad: el mundo subdesarrollado al que también pertenece América latina. La idea de que se pueden trazar paralelos entre fenómenos de la modemidad rusa y la latinoamericana ronda a los latinoamericanos desde hace un buen tiempo. Un ejemplo se encuentra en un texto del brasileño Roberto Schwarz, "As idéias fora do lugar', en A vencedoras batatas: Proceso social nos inicios d romance brasilero, Sao Paulo, Duas Cidade 1977. La reœpción de Bajtin puede también en tenderse dentro de esta idea. Una crítica importante a la visión de Beman es la de Ferry Anderso en "Modernidad y revolución". En Nicolás Casull (comp.). 92-116. Aquí se encuentra un cuestionamiento importante a la tendencia generalizada considerar que los modernismos corresponden un deteminado desarrollo económico y social y a más desarrollo más fértil será la producción simbólica.

${ }^{24}$ Berman, op. cit. Cita tomada de Casullo, op. oit.

${ }^{25}$ Nestor García Canclini. Culturas hibridas. 65.
} 
no se agota en el atraso». ${ }^{26}$ Sin embargo, no se trata aquí de la versión que José Joaquín Brunner llama la del "macondismo» ${ }^{27}$ y que hace eco a lo que describe Martín-Barbero como una idea que se manifiesta "pensando que la originalidad de los países latinoamericanos, y de América Latina en su conjunto, se halla constituida por factores que escapan a la lógica del desarrollo capitalista.» Por el contrario se trata de pensar que el atraso "ha sido históricamente producido"28 como de que la diferencia no se debe toda al atraso sino a la particular heterogeneidad cultural de los latinoamericanos.

El énfasis en los estudios culturales se debe a por lo menos dos factores decisivos. Por un lado está el lento reconocimiento del papel tan importante que des empeña lo cultural en la legitimación de las estructuras de poder. Por otro lado, el que lo cultural no se reduce ni a lo que las élites entienden por Cultura-literatura, arte, manifestaciones musicales cultas, etc., ni a lo que las instituciones culturales patrocinan y defienden, sino que cubre un espectro más amplio. El que se detecte una preocupación por similares cuestiones en diversos investigadores en las ciencias sociales no se debe a lo que siempre tiende a interpretarse en América Latina como una moda. Las circunstancias políticas de los años setenta condujeron entre otras a que muchos investigadores fueran al exilio intemo o externo. Esto permitió que se experimentara el continente de maneras nuevas, que se vieran realidades que permanecían ocultas. Pero también la complejidad de los fenómenos sociales ha generado la necesidad de otra lectura de nuestra modernidad, distanciándose también de la tendencia a señalar nuestra diferencia desde las carencias, de lo que nos falta o quedó faltando para ser como los europeos. ${ }^{29}$

Esto exige por supuesto un replanteamiento de las nociones de cultura para poder indicar desde dónde "lo cultural señala la percepción de dimensiones inéditas del conflicto social, la formación de nuevos sujetos -regionales, religiosos, sexuales, generacionales y formas nuevas de rebeldía y resistencia"30 pues otro de los fenómenos que se observa en los procesos sociales en América Latina es que muchas de esas fomas de "rebeldía y resistencia» se han dado en espacios distintos de aquellos que se contemplaban en los anális is tradicionales. No son ya los partidos políticos ni diversas instituciones desde donde se movilizan sino distintas formas de afiliación que van desde las comunidades religiosas, los movimientos artísticos, grupos que se cohesionan por diversos intereses. ${ }^{31}$ Los intereses de los distintos sujetos sociales se defienden desde diversas instancias, políticas, religiosas, sexuales. Para entender el significado y las implicaciones de estos fenómenos se hace necesario un desplazamiento del lugar desde donde se

\footnotetext{
${ }^{26}$ Jesús Martín-Barbero. De los medios a las mediaciones. Comunicación, cultura y hegemonía México: Editorial Gustavo Gui, 1987. 164.

27 José Joaquín Brunner. "La ciudad de los signos' En Op. oit. 37-72.

28 Op. cit. 165.

${ }^{29}$ Ver Brunner, op. cit. p.53 ss. Ver también los s gerentes ensayos de Aníbal Quijano. Modemida identidad y utopía en América Latina. Lima: Sociedad y Política, Ediciones. 1988.

${ }^{30}$ De los medios a las mediaciones, p. 226.

31 Ibid. P.228.
} 
plantean las preguntas, más allá de las ideologías y los intereses de ciertos grupos. $^{32}$

Igualmente importante dentro del debate es la incidencia que tienen los medios masivos de comunicación. Aquí el hito lo marca la obra de Jesús Martín-Barbero pues ha sido él quien más claramente ha señalado los puntos teóricos en los que se venía dando el debate sobre la cultura en América Latina y uno de los que ha entrado a cuestionarlos más a fondo. Su discusión sobre medios masivos pemite ver cómo se ha venido operando con nociones sobre la cultura popular y la masiva que no han permitido pensar las interpelaciones que la una hace desde la otra, los mestizajes que se producen. Igualmente muestra cómo los distintos discursos teóricos sobre lo masivo no permiten analizar los fenómenos masivos desde otro lugar de significación que para él cobra gran relevancia: el de los usos que se hacen de los medios y de los mensajes.

Su trabajo se mueve simultáneamente en tres planos: discute los presupuestos teóricos de las ciencias de la comunicación; señala las limitaciones que se presentan al estudiar un fenómeno social como es la comunicación sólo desde los medios, de las tecnologías, sin integrarlo a el ámbito más amplio de toda la interacción social; inscribe el anális is de lo masivo en la historia de los movimientos sociales y así ilumina un aspecto de la distinta conformación de la modernidad latinoamericana. El aspecto histórico es de particular relevancia en la medida en que al señalar el papel que han desempeñado los medios masivos en la constitución de las naciones y las identidades nacionales obligan a repensar muchos de los conceptos con los que se venía operando. Pues curiosamente en un continente con altísimos índices de analfabetismo, en el que la gran mayoría escucha radio y ve televisión pero no lee libros, se siguen pensando las identidades nacionales desde la cultura letrada, se consideran plasmadas en las grandes obras literarias.

En general, la discusión sobre las identidades es de gran relevancia y es uno de los aspectos en donde el pensamiento postmoderno tiene una incidencia significativa. Lo que ha caracterizado a los diversos discursos sobre la identidad en América Latina es la voluntad de fijar lo que sería substancialmente el carácter de una nación, una región o todo el subcontinente. El pensamiento posmodemo se distancia de esta forma substancialista de concebir las identidades y permite repensar éstas no des de lo fijo, lo inmutable, sino desde lo dinámico y cambiante. Este desplazamiento hace posible igualmente pensar la representatividad democrática no como la fijación de cuotas para grupos perfectamente definidos dentro de una sociedad, sino desde las dinámicas de negociación constante. Así lo propone, por ejemplo, García Canclini al señalar cómo «captamos muy poco del

\footnotetext{
${ }^{32}$ Ver del politólogo chileno Norbent Lechner, La conflictiva y nunca acabada $\infty$ nstrucción del orden deseado, Madrid: CIS y Siglo XXI, 1986; 'Un desencanto llamado posmodemo ', en Punto de vista, XI, Nr. 33, 1988, p. 25-31; "La democratización en el contexto de una cultura posmodema", en Herlinghaus, Walter, eds. p. 197-209.
} 
poder si sólo registramos los enfrentamientos y las acciones verticales» ${ }^{33}$ La heterogeneidad cultural de América Latina no implica únicamente la existencia de diversas culturas, sino sobre todo la hibridación de éstas, lo que genera permanentes desplazamientos.

La teoría de la cultura en América Latina si bien plantea el debate des de un ámbito académico y de intelectuales, busca nuevas fomas de aproximarse a la praxis social. Ante el fracaso de las izquierdas, el fin de las utopías, las políticas económicas neoliberales, las maneras diversas en que se manifiestan los movimientos sociales y los nuevos interrogantes que plantean los medios transnacionales de comunicación el desafío está en repensar esa modernidad que se postulaba como aquello que había que lograr a toda costa, así fuera sacrificando los intereses de las mayorías.

Ahora bien, las grandes teorías, los metarrelatos, hacían de esas mayorías un todo unificado, las presentaban como algo homogéneo. Lo que evidencian los análisis de estos autores es que la fragmentación, la diversidad no es necesariamente lo que hay que superar, sino el punto desde donde hay que partir para buscar los téminos de las negociaciones que una verdadera democracia exige. Permiten pensar la diferencia, lo cual no debe significar una justificación de la desigualdad social y económica.

Dentro de los debates sobre la pos-modemidad se oscila entre un extremo que afirma que América Latina siempre ha sido posmoderna y otro que niega la posmodernidad en un continente que ni siquiera ha logrado ser modemo. Ambas tendencias olvidan que no se trata de ver cómo se puede inscribir a América Latina dentro del discurso de moda. El pensamiento posmodemo abre la posibilidad de pensar sobre nuestra compleja realidad cultural, social y económica. Pero no es el único, ni es la única manera de hacerlo. América Latina tiene una larga tradición de pensamiento que no se está echando por la borda, sino reestructurando, desplazando. No se trata aquí de generar un pensamiento que sea exclusivamente latinoamericano (en caso de que tal cosa fuera deseable o posible), sino de bus car cómo pensar nuestros conflictos en un momento histórico de globalización económica y fragmentación social. Lo que tiene de propio este pensamiento es que se refiere a nuestros problemas. No me parece relevante establecer si las ideas con las que se opera surgieron aquí o allá. Se trata más bien de hacer uso de todas las que pemitan acercarse a los problemas; las circunstancias propias de los latinoamericanos, de los colombianos, reubican estas ideas, las desplazan, se da un extrañamiento de su contexto original y una apropiación en el nuevo contexto. Es la forma como se ha venido elaborando el pensamiento en nuestro continente desde siempre.

No se trata de ser original en el plano de las ideas, sino más bien de buscar caminos para una sociedad más justa. Pues si bien es evidente que las utopías de

${ }^{33}$ Culturas híbridas, p. 324. 
la modemidad, ancladas a una foma de pensamiento universalista y totalitarista, se han agotado, eso no significa necesariamente una renuncia a buscar un futuro mejor para todos. La cuestión está en cómo hacerlo. Es evidente que los Estados y el neoliberalismo no han renunciado a la totalidad y que el consumo amenaza con convertirse en la ley de consenso universal. Ante esto una crítica social no substancialista amenaza con caer en el relativismo y parece ser muy débil para enfrentarse a los problemas. El lugar desde donde se hace esta crítica ya no es tan claro, hay que definir las posiciones constantemente, pero nunca había sido más necesaria.

La lógica de la economía por lado, y la crisis ideológica, por el otro, hacen sentir cada vez mas fuertemente que ciencias sociales y las humanidades perdieron toda relevancia. Para que una forma de pensar que evita los dogmatismos no termine haciéndole el juego a un sistema económico que sabe aprovechar muy bien las inseguridades políticas no se nos puede olvidar que, como dice García Canclini, «trata de averiguar... cómo ser radical sin ser fundamentalista». ${ }^{34}$ Es en estos momentos que se hace vital repensar nuestra modernidad, las nociones de progreso lineal que se nos ofrece desde la economía, un progreso que hace pagar el precio a lo social pasa por alto la diversidad, heterogeneidad y complejidad de las culturas. Las aperturas del pensamiento posmoderno pueden ser una ayuda para romper con el dualismo en el que movían los debates, buscar caminos ternativos, terceras vías.

\footnotetext{
34 Ibid. p. 348. Aquí me he basado en varios textos, además del de García Candini. Ver: Martín Barbero (1992), Beatriz Sano (1994>, Homi Bhabha (1994). Adicionalmente quiero agradecer en este punto a los miembros del Grupo de Pensamiento Latinoamericano de la Universidad de Tübingen, Alemania que me invitó a discutir estos temas con ellos. Una ayuda para organizar estas ideas ha sido el diálogo con Santiago Castro Gómez y su texto La dialéctica de la modernidad en América Latina:
} 